\title{
Prevalence and risk factors of undernutrition among antiretroviral-therapy-naïve subjects aged under 5 years old in Makurdi, Nigeria: a retrospective study
}

This article was published in the following Dove Press journal:

International Journal of General Medicine

3I March 2015

Number of times this article has been viewed

\author{
Emmanuel Ademola \\ Anigilaje \\ Ayodotun Olutola ${ }^{2}$ \\ 'Department of Paediatrics, Benue \\ State University, Makurdi, ${ }^{2}$ Center \\ for Clinical Care and Clinical \\ Research, Abuja, Nigeria
}

Correspondence: Emmanuel Ademola Anigilaje

Department of Paediatrics, Benue State University, PMB 102119, Makurdi, Benue State, Nigeria

Tel +2348033833839

Email demolaanigilaje@yahoo.co.uk
Background: Undernutrition is common in human immunodeficiency virus (HIV) infection and it contributes significantly to its morbidity and mortality. However, as far as we are aware, few studies have described the risk factors of undernutrition among HIV-infected Nigerian children. The study reported here aimed to determine the prevalence and risk factors of undernutrition among HIV-infected, antiretroviral therapy (ART)-naïve children aged under 5 years old in Makurdi, Nigeria.

Methods: A retrospective, cross-sectional study was undertaken at the Federal Medical Centre, Makurdi, between June 2010 and June 2011. Logistic regression modelling was used to determine the risk factors of undernutrition.

Results: Data on 182 HIV-infected children ( 88 males and 94 females), aged between 6 weeks and 59 months were studied. The prevalence of undernutrition was $12.1 \%, 33.5 \%$, and $54.4 \%$ for underweight, wasting, and stunting, respectively. In multivariate regression analyses, being female (adjusted odds ratio [AOR] 0.292, 95\% [confidence interval] CI 0.104-0.820, $P=0.019$ ), the child's caregiver being on ART (AOR 0.190, 95\% CI 0.039-0.925, $P=0.04$ ), and the absence of tuberculosis in the child (AOR $0.034,95 \%$ CI $0.003-0.357, P=0.005$ ) were independently protective against underweight. Subjects who were exclusively breastfed in the first 6 months of life were protected from stunting (AOR $0.136,95 \%$ CI $0.032-0.585, P=0.007$ ). No factor impacted significantly on wasting in multivariate analyses.

Conclusion: Undernutrition among HIV-infected, ART-naïve children aged under 5 years old may be reduced if programmatic interventions are guided toward early initiation of ART among eligible HIV-infected caregivers and the promotion of HIV/tuberculosis coinfection control efforts. Also, the importance of exclusive breastfeeding in reducing undernutrition cannot be overemphasized.

Keywords: HIV infection, ART, HIV/tuberculosis coinfection, stunting, breastfeeding, mother-to-child transmission

\section{Introduction}

Regional differences in the HIV burden exist in Nigeria. ${ }^{1}$ The ANC HIV Sentinel Survey of pregnant women in antenatal care in Nigeria revealed that Benue State, located in the North Central region, has the highest human immunodeficiency virus (HIV) prevalence rates $-10 \%$ in $2005,10.6 \%$ in 2008 , and $12.7 \%$ in $2010 .{ }^{1}$ Reasons adduced for the high HIV prevalence in Benue State include the high level of poverty and illiteracy, some sociocultural practices, and the lack of financial empowerment for women. ${ }^{2}$ Unfortunately, $80 \%$ of HIV-infected pregnant women in Nigeria did not receive antiretroviral medicines for the prevention of mother-to-child transmission of HIV in $2012,{ }^{3}$ and with the mother-to-child transmission rate as high as $34 \%$ in our 
own setting, ${ }^{4}$ the prospects of achieving a reduction in the burden of perinatal HIV infection appear bleak.

In 2008, the prevalence of undernutrition among Nigerian children aged under 5 years old in the general population was $41 \%, 23 \%$, and $14 \%$ for stunting, underweight, and wasting, respectively, ${ }^{5}$ and $11 \%$ of children younger than 15 years old were also infected with HIV in the same year. ${ }^{6}$

Complex interactions exist between HIV infection and growth/nutrition, such that children with HIV infection are prone to undernutrition, ${ }^{7-11}$ and mortality from severe acute malnutrition is more than three-times higher in HIV-positive children than their HIV-negative counterparts. ${ }^{9}$ In addition, undernutrition also results in an impaired response to ART in HIV infection. ${ }^{12}$

The mechanism for growth failure in HIV infection involves inadequate caloric intake, gastrointestinal infestations, opportunistic infections, abnormal resting energy expenditure, endocrine abnormalities, and HIV enteropathy. ${ }^{10,13-15}$

A review of the literature reveals that the prevalence of undernutrition among HIV-infected ART-naïve Nigerian children was $11.9 \%$ in Enugu, South Eastern region in 2005; ${ }^{16}$ $58.6 \%$ in Ile-Ife, South Western region (2007-2009); ${ }^{17}$ and $77.3 \%$ in Kano, Northern region in $2010 .{ }^{18} \mathrm{~A}$ high burden of undernutrition of $55 \%$ and $56.4 \%$ was also reported among HIV-infected ART-naïve children in Cameroon (2008-2009) ${ }^{19}$ and India (2004-2006), respectively. ${ }^{20}$

As far as we are aware, the study reported here is the first to describe the burden of undernutrition among HIV-infected ART-naïve children in Makurdi, a city with a high burden of perinatal HIV infection in North Central Nigeria. ${ }^{1}$ This study is important because undernutrition, regardless of its etiology, contributes to under-5 mortality, ${ }^{5}$ impairs immunity and predisposes a child to infectious illnesses, and also impairs the child's motor and cognitive development. ${ }^{21}$

Table I Age, sex, and undernutrition distribution among the 182 subjects

\begin{tabular}{ll}
\hline Characteristic & Number (\%) \\
\hline Age (months) & \\
$0-23$ & $34(18.7)$ \\
$24-59$ & $148(81.3)$ \\
Sex & \\
Male & $88(48.4)$ \\
Female & $94(51.6)$ \\
Undernutrition (not mutually exclusive) & \\
Underweight & $22(12.1)$ \\
Wasting & $61(33.5)$ \\
Stunting & $99(54.4)$ \\
No undernutrition & $69(37.9)$ \\
\hline
\end{tabular}

The need for this study was also strengthened by the fact that although highly active antiretroviral therapy (HAART) has been shown to improve the nutritional status of HIV-infected children by some studies, ${ }^{22,23}$ a Tanzanian study ${ }^{24}$ also found that HAART alone is not enough to resolve the nutritional problems in HIV-infected children, underscoring the need for a more robust specific nutritional therapy. Moreover, in Nigeria, very few (less than $12 \%$ ) children in need of HAART were receiving it, in 2012. ${ }^{25}$

Although knowledge about the growth response of our HIV-infected children to HAART is important and also being studied by the authors, the present study focuses on the prevalence of undernutrition and its risk factors among ART-naïve HIV-infected children at enrollment into an ART program. We expect programmatic nutritional interventions to be better guided if the risk factors of undernutrition are well elucidated, especially among our HIV-infected children who require nutritional interventions, either alone in care or in conjunction with HAART in treatment.

\section{Materials and methods Study design}

This was a retrospective cross-sectional study among HIVinfected ART-naïve children.

\section{Study area}

The study took place between June 2010 and June 2011 at the Paediatric ART Clinic of the Federal Medical Centre (FMC), Makurdi, Benue State, Nigeria. The facility is supported by the AIDS Prevention Initiative in Nigeria (APIN)/Harvard [US] President's Emergency Program for AIDS Relief (PEPFAR). Benue State lies within the middle belt region of Nigeria. The state has a population of over 5 million people and occupies a landmass of 34,059 square kilometers. The state experiences two distinct seasons, the wet/rainy season and the dry/summer season. The rainy season, during which farming takes place, lasts from April to October, with an annual rainfall in the range of 100-200 mm. The dry season begins in November and ends in March. Temperatures fluctuate between $23^{\circ} \mathrm{C}$ and $37^{\circ} \mathrm{C}$.

\section{Ethical considerations}

Upon enrollment into care, all parents or caregivers of the HIVinfected children were required to provide written informed consent (and assent from the children if aged $\geq 7$ years) for the use of their data for research as approved by the Hospital Research and Ethics Committee (HREC) of the FMC, Makurdi, and the APIN/Harvard PEPFAR. For this study, 
Table 2 Prevalence and risk factors of underweight among subjects aged under 5 years old

\begin{tabular}{|c|c|c|c|c|c|c|c|c|}
\hline \multirow[t]{2}{*}{ Clinical variable } & \multirow{2}{*}{$\begin{array}{l}\text { Underweight } \\
\text { (WAZ-score }<-2 S D \text { ) } \\
\text { N (\%) }\end{array}$} & \multirow{2}{*}{$\begin{array}{l}\text { Not underweight } \\
\text { (WAZ-score } \geq-2 S D \text { ) } \\
\text { N (\%) }\end{array}$} & \multicolumn{3}{|c|}{ Bivariate analysis } & \multicolumn{3}{|c|}{ Multivariate analysis } \\
\hline & & & OR & $95 \% \mathrm{Cl}$ & $P$-value & AOR & $95 \% \mathrm{Cl}$ & $P$-value \\
\hline \multicolumn{9}{|l|}{ Demography } \\
\hline \multicolumn{9}{|l|}{ Age (months) } \\
\hline Median & 2.00 & 3.00 & - & - & 0.033 & - & - & - \\
\hline $0-23$ & $16(26.2)$ & $18(14.9)$ & 2.035 & $0.952-4.346$ & 0.064 & 0.706 & $0.204-2.437$ & 0.581 \\
\hline 24-59 (ref) & $45(73.8)$ & $103(85.1)$ & & & & & & \\
\hline \multicolumn{9}{|l|}{ Sex } \\
\hline Male & $36(59.0)$ & $52(43.0)$ & 1.911 & I.1023-3.568 & $0.04 I$ & 0.292 & $0.104-0.820$ & 0.019 \\
\hline Female (ref) & $25(41.0)$ & $69(57.0)$ & & & & & & \\
\hline \multicolumn{9}{|l|}{ Orphanhood } \\
\hline Yes & $22(36.1)$ & $27(22.3)$ & 1.964 & $0.999-3.859$ & 0.048 & 1.224 & $0.285-5.248$ & 0.786 \\
\hline No (ref) & $39(63.9)$ & $94(77.7)$ & & & & & & \\
\hline \multicolumn{9}{|c|}{ Family/socioeconomic factors } \\
\hline \multicolumn{9}{|c|}{ Mode of HIV transmission } \\
\hline Vertical & $61(100.0)$ & I0I (83.5) & 0.623 & $0.553-0.703$ & 0.001 & 0.000 & - & 1.000 \\
\hline Blood transfusion (ref) & $0(0.0)$ & $20(16.5)$ & & & & & & \\
\hline \multicolumn{9}{|l|}{ Mode of infant feeding } \\
\hline EBMS & $39(63.9)$ & $42(34.7)$ & - & - & 0.000 & 6.902 & & 0.998 \\
\hline MF & $3(4.9)$ & $75(62.0)$ & & & & 1.005 & - & 0.998 \\
\hline EBF (ref) & $19(31.1)$ & $4(3.3)$ & & & & & - & \\
\hline \multicolumn{9}{|l|}{ Caregivers' HIV status } \\
\hline Infected & $61(100.0)$ & $103(85.1)$ & 0.628 & $0.558-0.707$ & 0.002 & - & - & - \\
\hline Not infected (ref) & $0(0.0)$ & $18(14.9)$ & & & & & & \\
\hline \multicolumn{9}{|l|}{ Caregiver on HAART } \\
\hline No & $20(32.8)$ & $8(7.8)$ & 5.793 & $2.360-14.219$ & 0.000 & 0.190 & $0.039-0.925$ & 0.040 \\
\hline Yes (ref) & $4 \mid(67.2)$ & $95(92.2)$ & & & & & & \\
\hline \multicolumn{9}{|c|}{ Marital status of caregiver } \\
\hline Without partner & $16(26.2)$ & $9(7.4)$ & 4.425 & $|.823-| 0.74 \mid$ & 0.001 & 0.134 & $0.017-1.028$ & 0.053 \\
\hline With a partner (ref) & $45(73.8)$ & $112(92.6)$ & & & & & & \\
\hline \multicolumn{9}{|c|}{ Number of people in the household } \\
\hline$>5$ & $37(60.7)$ & $59(48.8)$ & 1.620 & $0.867-3.027$ & 0.129 & - & - & - \\
\hline$\leq 5$ (ref) & $24(39.3)$ & $62(5 \mid .2)$ & & & & & & \\
\hline Place of residence & & & & & & & & \\
\hline Urban & $9(14.8)$ & $15(12.4)$ & 1.223 & $0.502-2.980$ & 0.657 & - & - & - \\
\hline Rural (ref) & $52(85.2)$ & $106(87.6)$ & & & & & & \\
\hline Clinical/laboratory finc & ings & & & & & & & \\
\hline WHO clinical staging & & & & & & & & \\
\hline 3 and 4 & $29(47.5)$ & $20(16.5)$ & 4.577 & $2.285-9.167$ & 0.000 & 2.818 & $0.355-22.367$ & 0.327 \\
\hline $\mathrm{I}$ and 2 (ref) & $32(52.5)$ & I0I (83.5) & & & & & & \\
\hline CD4 count & & & & & & & & \\
\hline Median (IQR) & $552.50(268.0-938.75)$ & - & - & & 0.31 & - & - & - \\
\hline$\leq 200$ & I5 (24.6) & $17(14.0)$ & 1.995 & $0.918-4.335$ & 0.078 & 0.698 & $0.196-2.481$ & 0.578 \\
\hline$>200$ (ref) & $46(75.4)$ & $104(86.0)$ & & & & & & \\
\hline Viral load (copies/mL) & & & & & & & & \\
\hline Median log 10 (IQR) & $4.66(3.7|-5.3|)$ & - & - & - & 0.237 & - & - & - \\
\hline$>10,000$ & $43(70.5)$ & $81(66.9)$ & 1.180 & $0.605-2.301$ & 0.628 & - & - & - \\
\hline$<10,000$ (ref) & I8 (29.5) & $40(33.1)$ & & & & & & \\
\hline Hepatitis B surface antige & & & & & & & & \\
\hline Yes & $7(11.5)$ & II (9.I) & 1.296 & $0.476-3.531$ & 0.611 & - & - & - \\
\hline No (ref) & $54(88.5)$ & $110(90.9)$ & & & & & & \\
\hline Hepatitis $C$ antibodies & & & & & & & & \\
\hline Yes & $2(3.3)$ & $3(2.5)$ & 1.322 & $0.215-8.130$ & 1.000 & - & - & - \\
\hline No (ref) & $59(96.7)$ & $117(97.5)$ & & & & & & \\
\hline Diagnosed comorbidit & es/opportunistic infectic & & & & & & & \\
\hline Tuberculosis & & & & & & & & \\
\hline Yes & $25(4 \mid .0)$ & $12(9.9)$ & 6.308 & $2.878-13.825$ & 0.000 & 0.034 & $0.003-0.357$ & 0.005 \\
\hline No (ref) & $36(59.0)$ & $109(90.1)$ & & & & & & \\
\hline
\end{tabular}


Table 2 (Continued)

\begin{tabular}{|c|c|c|c|c|c|c|c|c|}
\hline \multirow[t]{2}{*}{ Clinical variable } & \multirow{2}{*}{$\begin{array}{l}\text { Underweight } \\
\text { (WAZ-score < -2SD) } \\
\text { N (\%) }\end{array}$} & \multirow{2}{*}{$\begin{array}{l}\text { Not underweight } \\
\text { (WAZ-score } \geq-2 S D \text { ) } \\
\text { N (\%) }\end{array}$} & \multicolumn{3}{|c|}{ Bivariate analysis } & \multicolumn{3}{|c|}{ Multivariate analysis } \\
\hline & & & OR & $95 \% \mathrm{Cl}$ & $P$-value & AOR & $95 \% \mathrm{Cl}$ & $P$-value \\
\hline \multicolumn{9}{|c|}{ Oropharyngeal candidiasis } \\
\hline Yes & $9(14.8)$ & $9(7.4)$ & 2.154 & $0.808-5.743$ & 0.119 & - & - & - \\
\hline No (ref) & $52(85.2)$ & $112(92.6)$ & & & & & & \\
\hline \multicolumn{9}{|c|}{ Esophageal candidiasis } \\
\hline Yes & $4(6.6)$ & $\mathrm{I}(0.8)$ & 8.421 & $0.920-77.062$ & 0.080 & 0.233 & $0.009-6.174$ & 0.383 \\
\hline No (ref) & $57(93.4)$ & $120(99.2)$ & & & & & & \\
\hline \multicolumn{9}{|l|}{ Diarrheal disease } \\
\hline Yes & $2(3.3)$ & $5(4.1)$ & 0.786 & $0.148-4.176$ & 0.777 & - & - & - \\
\hline No (ref) & $59(96.7)$ & $116(95.9)$ & & & & & & \\
\hline \multicolumn{9}{|l|}{ Sepsis } \\
\hline Yes & I (I.6) & $8(6.6)$ & 0.235 & $0.029-1.927$ & 0.272 & - & - & - \\
\hline No (ref) & $60(98.4)$ & $113(93.4)$ & & & & & & \\
\hline \multicolumn{9}{|l|}{ Sickle-cell disease } \\
\hline Yes & $3(4.9)$ & $6(5.0)$ & 0.991 & $0.239-4.107$ & 0.990 & - & - & - \\
\hline No (ref) & $58(95.1)$ & II $5(95.0)$ & & & & & & \\
\hline \multicolumn{9}{|l|}{ Pneumonia } \\
\hline Yes & $3(4.9)$ & $5(4.1)$ & 1.200 & $0.277-5.196$ & 0.807 & - & - & - \\
\hline No (ref) & $58(95.1)$ & 116 (95.9) & & & & & & \\
\hline \multicolumn{9}{|l|}{ Anemia $(<8 \mathrm{~g} / \mathrm{dL})$} \\
\hline Yes & $40(65.6)$ & $69(57.0)$ & 0.697 & $0.368-1.320$ & 0.267 & - & - & - \\
\hline No & $21(34.4)$ & $52(43.0)$ & & & & & & \\
\hline
\end{tabular}

Abbreviations: AOR, adjusted odds ratio; $\mathrm{Cl}$, confidence interval; EBF, exclusive breast feeding; EBMS, exclusive breast-milk-substitute feeding; HAART, highly active antiretroviral therapy; IQR, interquartile range; MF, mixed feeding; N, number; OR, odds ratio; Ref, reference group; SD, standard deviation; WAZ-score, weight-for-age Z-score; WHO, World Health Organization.

permission was sought for and received from the HREC of the FMC for the use of the relevant data.

\section{Inclusion criteria}

Included in the study were HIV-infected children ( $\leq 15$ years of age) who were ART naïve and whose anthropometrical growth parameters and records of potential risk factors of undernutrition were available on enrollment into our ART program.

\section{Recruitment of subjects and data collection}

FMC, Makurdi, provides pediatric HIV care and treatment in accordance with the Nigerian guidelines on pediatric HIV/AIDS treatment and care. ${ }^{25,26}$ Children were recruited into care and treatment if they were confirmed to be HIV infected. All subjects aged $\geq 18$ months had initial doublerapid HIV antibody tests using Determine ${ }^{\mathrm{TM}} \mathrm{HIV}-1 / 2$ first and then HIV $1 / 2$ STAT-PAK ${ }^{\circledR}$. HIV infection was confirmed in those with a reactive rapid test by the Western blot test. Two HIV DNA polymerase-chain-reaction positivity tests for those aged $<18$ months confirmed HIV infection in this age group.

A study proforma was developed to capture the following information that had been recorded on each subject's initial clinical evaluation form at enrollment into our program: mode of HIV transmission, caregiver HIV status, whether caregiver was on ART, caregiver marital status, place of residence (rural vs urban), number of people in the household, whether the child was an orphan, mode of infant feeding, HIV/AIDS clinical stage, anthropometric measurements, and other diagnosed comorbidities/opportunistic infections (tuberculosis, oropharyngeal and esophageal candidiasis, diarrheal disease, presumed sepsis, malaria fever, pneumonia, and hepatitis B and hepatitis $\mathrm{C}$ viral infections).

\section{Operational definitions}

For the purpose of the study, the following terms were defined. "Exclusive breastfeeding" (EBF) was defined as the infant receiving only breast milk for the first 6 months of life from his/her mother and no other liquids or solids, with the exception of drops or syrups consisting of vitamins, mineral supplements, or drugs. ${ }^{27}$ "Exclusive breast-milk-substitute feeding" (EBMS) was defined as the administration of infant formula for the first 6 months of life and the exclusion of all breastfeeding. ${ }^{27}$ "Mixed feeding" (MF) was defined as giving breast milk with breast-milk substitute or other liquids or solid foods; ${ }^{27}$ two episodes of instances of mixed feeding were required for this definition to apply. 
Table 3 Prevalence and risk factors of wasting among subjects aged under 5 years old

\begin{tabular}{|c|c|c|c|c|c|c|c|c|}
\hline \multirow[t]{2}{*}{ Clinical variable } & \multirow{2}{*}{$\begin{array}{l}\text { Wasted } \\
\text { (WHZ-score }<-2 \text { SD) } \\
\text { N (\%) }\end{array}$} & \multirow{2}{*}{$\begin{array}{l}\text { Not wasted } \\
\text { (WHZ-score } \geq-2 \text { SD) } \\
\text { N (\%) }\end{array}$} & \multicolumn{3}{|c|}{ Bivariate analysis } & \multicolumn{3}{|c|}{ Multivariate analysis } \\
\hline & & & OR & $95 \% \mathrm{Cl}$ & $P$-value & AOR & $95 \% \mathrm{Cl}$ & $P$-value \\
\hline \multicolumn{9}{|l|}{ Demography } \\
\hline \multicolumn{9}{|l|}{ Age (months) } \\
\hline Median & 2.00 & 3.00 & - & - & 0.022 & - & - & - \\
\hline $0-23$ & $8(36.4)$ & $26(16.3)$ & 2.945 & I. $122-7.729$ & 0.048 & 0.362 & $0.042-3.082$ & 0.352 \\
\hline 24-59 (ref) & $14(63.6)$ & $134(83.8)$ & & & & & & \\
\hline \multicolumn{9}{|l|}{ Sex } \\
\hline Male & I $3(59.1)$ & 75 (46.9) & & & & & & \\
\hline Female (ref) & $9(40.9)$ & $85(53.1)$ & 1.637 & $0.662-4.046$ & 0.282 & - & - & - \\
\hline \multicolumn{9}{|l|}{ Orphanhood } \\
\hline Yes & II (50.0) & $38(23.8)$ & 3.211 & I.290-7.989 & 0.009 & 7.939 & - & 0.998 \\
\hline No (ref) & II (50.0) & $122(76.3)$ & & & & & & \\
\hline \multicolumn{9}{|c|}{ Family/socioeconomic factors } \\
\hline \multicolumn{9}{|c|}{ Mode of HIV transmission } \\
\hline Vertical & $22(100.0)$ & $140(87.5)$ & 0.864 & $0.813-0.919$ & 0.079 & - & - & - \\
\hline Blood & $0(0.0)$ & $20(12.5)$ & & & & & & \\
\hline transfusion (ref) & & & & & & & & \\
\hline \multicolumn{9}{|l|}{ Mode of infant feeding } \\
\hline EBMS & $4(18.2)$ & $77(48.1)$ & - & - & 0.000 & 1.125 & - & 0.996 \\
\hline MF & $0(0.0)$ & $78(48.8)$ & & & & 4.524 & - & 0.995 \\
\hline EBF (ref) & I8 (8।.8) & $5(3.1)$ & & & & & & \\
\hline \multicolumn{9}{|l|}{ Caregivers' HIV Status } \\
\hline Infected & $22(100.0)$ & $142(88.8)$ & 0.866 & $0.815-0.920$ & 0.097 & - & - & - \\
\hline Not infected (ref) & $0(0.0)$ & $18(I I .3)$ & & & & & & \\
\hline \multicolumn{9}{|l|}{ Caregiver on HAART } \\
\hline No & $12(54.5)$ & $16(11.3)$ & 9.450 & $3.521-25.365$ & 0.000 & 1.706 & $0.117-24.960$ & 0.696 \\
\hline Yes (ref) & $10(45.5)$ & $126(88.7)$ & & & & & & \\
\hline \multicolumn{9}{|c|}{ Marital status of caregiver } \\
\hline Without partner & II (50.0) & $14(8.8)$ & 10.429 & $3.838-28.336$ & 0.000 & 0.000 & - & 0.998 \\
\hline With a partner (ref) & II (50.0) & $146(91.3)$ & & & & & & \\
\hline Number of people in th & e household & & & & & & & \\
\hline$>5$ & $15(68.2)$ & $81(50.6)$ & 2.090 & $0.809-5.400$ & 0.122 & - & - & - \\
\hline$\leq 5$ (ref) & $7(3 \mid .8)$ & $79(49.4)$ & & & & & & \\
\hline Place of residence & & & & & & & & \\
\hline Urban & $2(9.1)$ & $22(13.8)$ & 0.627 & $0.137-2.873$ & 0.545 & - & - & - \\
\hline Rural (ref) & $20(90.9)$ & $138(86.3)$ & & & & & & \\
\hline Clinical/laboratory fi & ndings & & & & & & & \\
\hline WHO clinical staging & & & & & & & & \\
\hline 3 and 4 & $16(72.7)$ & $33(20.6)$ & 10.263 & $3.725-28.273$ & 0.000 & 2.173 & $0.000-10,622.45$ & 0.858 \\
\hline I and 2 (ref) & $6(27.3)$ & $127(79.4)$ & & & & & & \\
\hline CD4 count & & & & & & & & \\
\hline Median (IQR) & $552.50(268.00-938.75)$ & - & - & - & 0.025 & - & - & - \\
\hline$\leq 200$ & $8(36.4)$ & $24(15.0)$ & 3.238 & $|.226-8.55|$ & 0.014 & 0.890 & $0.093-8.556$ & 0.919 \\
\hline$>200$ (ref) & $14(63.6)$ & $136(85.0)$ & & & & & & \\
\hline Viral load (copies/mL) & & & & & & & & \\
\hline Median log I0 (IQR) & $4.66(3.716-5.315)$ & - & - & - & 0.516 & - & - & - \\
\hline$>10,000$ & $13(59.1)$ & III (69.4) & 0.638 & $0.256-1.590$ & 0.332 & - & - & - \\
\hline$<10,000$ (ref) & $9(40.9)$ & $49(30.6)$ & & & & & & \\
\hline Hepatitis B surface antį & & & & & & & & \\
\hline Yes & $4(18.2)$ & $14(8.8)$ & 2.317 & $0.688-7.805$ & 0.313 & - & - & - \\
\hline No (ref) & I8 (8I.8) & $146(91.3)$ & & & & & & \\
\hline Hepatitis $C$ antibodies & & & & & & & & \\
\hline Yes & $0(0.0)$ & $5(3.1)$ & 1.143 & $1.08 \mathrm{I}-1.208$ & 0.399 & - & - & - \\
\hline No (ref) & $22(100.0)$ & $154(96.9)$ & & & & & & \\
\hline Diagnosed comorbid & ities/opportunistic infec & tions & & & & & & \\
\hline Tuberculosis & & & & & & & & \\
\hline Yes & $14(63.6)$ & $23(14.4)$ & 10.424 & $3.934-27.621$ & 0.000 & 0.067 & $0.000-375.863$ & 0.539 \\
\hline No (ref) & $8(36.4)$ & $137(85.6)$ & & & & & & \\
\hline
\end{tabular}


Table 3 (Continued)

\begin{tabular}{|c|c|c|c|c|c|c|c|c|}
\hline \multirow[t]{2}{*}{ Clinical variable } & \multirow{2}{*}{$\begin{array}{l}\text { Wasted } \\
\text { (WHZ-score }<-2 \text { SD) } \\
\text { N (\%) }\end{array}$} & \multirow{2}{*}{$\begin{array}{l}\text { Not wasted } \\
\text { (WHZ-score } \geq-2 \text { SD) } \\
\text { N (\%) }\end{array}$} & \multicolumn{3}{|c|}{ Bivariate analysis } & \multicolumn{3}{|c|}{ Multivariate analysis } \\
\hline & & & OR & $95 \% \mathrm{Cl}$ & $P$-value & AOR & $95 \% \mathrm{Cl}$ & $P$-value \\
\hline \multicolumn{9}{|c|}{ Oropharyngeal candidiasis } \\
\hline Yes & $2(9.1)$ & $16(10.0)$ & 0.900 & $0.192-4.209$ & 1.000 & - & - & - \\
\hline No (ref) & $20(90.9)$ & $144(90.0)$ & & & & & & \\
\hline \multicolumn{9}{|c|}{ Esophageal candidiasis } \\
\hline Yes & I (4.5) & $4(2.5)$ & 1.857 & $0.198-17.413$ & 1.000 & - & - & - \\
\hline No (ref) & $21(95.5)$ & $156(97.5)$ & & & & & & \\
\hline \multicolumn{9}{|l|}{ Diarrheal disease } \\
\hline Yes & $0(0.0)$ & $7(4.4)$ & 1.144 & $1.081-1.210$ & 0.682 & - & - & - \\
\hline No (ref) & $22(100.0)$ & $153(95.6)$ & & & & & & \\
\hline \multicolumn{9}{|l|}{ Sepsis } \\
\hline Yes & $4(18.2)$ & $5(3.1)$ & 6.889 & $1.695-28.004$ & 0.011 & 0.550 & $0.000-2,425.15$ & 0.889 \\
\hline No (ref) & $18(81.8)$ & $155(96.9)$ & & & & & & \\
\hline \multicolumn{9}{|l|}{ Sickle-cell disease } \\
\hline Yes & $3(13.6)$ & $6(3.8)$ & 4.053 & $0.936-17.548$ & 0.139 & - & - & - \\
\hline No (ref) & $19(86.4)$ & $154(96.3)$ & & & & & & \\
\hline \multicolumn{9}{|l|}{ Pneumonia } \\
\hline Yes & I (4.5) & $7(4.4)$ & 1.041 & $0.122-8.885$ & 1.000 & - & - & - \\
\hline No (ref) & $21(95.5)$ & $153(95.6)$ & & & & & & \\
\hline \multicolumn{9}{|l|}{ Anemia $(<8 \mathrm{~g} / \mathrm{dL})$} \\
\hline Yes & $15(68.2)$ & $94(58.8)$ & 0.665 & $0.257-1.720$ & 0.397 & - & - & - \\
\hline No & $7(31.8)$ & $66(4 I .3)$ & & & & & & \\
\hline
\end{tabular}

Abbreviations: AOR, adjusted odds ratio; $\mathrm{Cl}$, confidence interval; EBF, exclusive breast feeding; EBMS, exclusive breast-milk-substitute feeding; HAART, highly active antiretroviral therapy; IQR, interquartile range; MF, mixed feeding; N, number; OR, odds ratio; Ref, reference group; SD, standard deviation; WHZ-score, weight-for-height Z-score; WHO, World Health Organization.

To define "undernutrition" in children aged less than 5 years old, anthropometric computations and comparisons were conducted using World Health Organization (WHO) Anthro software (version 2.0, 2008), which is based on WHO child-growth standards of 2006. "28 "Underweight" was defined as a weight-for-age Z-score (WAZ-score) less than-2 standard deviations (SD) from the reference median values. "Stunting" was defined as a height-for-age Z-score (HAZscore) of less than -2SD from the reference median values. A weight-for-height Z-score (WHZ-score) less than -2SD from the reference median defined "wasting".

The definitions of "diarrhea", "chronic diarrhea", "pneumonia", "tuberculosis", "oropharyngeal candidiasis", "esophageal candidiasis", and "sepsis" used were those described in the WHO Pocket Book of Hospital Care for Children: Guidelines for the Management of Common Childhood Illnesses. ${ }^{29}$

\section{Laboratory measurements}

The cluster of differentiation 4 (CD4) count and the viral load of the subjects were determined at enrollment. Venous blood samples were also collected for malaria parasites (Giemsa stain), hepatitis B surface antigen, and hepatitis $C$ virus antibody (the third-generation enzyme-linked immunosorbent assay technique was used for hepatitis B surface antigen and hepatitis C virus antibody). All tests were done at the APIN/ PEPFAR laboratory of FMC, Makurdi.

\section{Statistical analysis}

Descriptive statistics were tabulated as medians for continuous variables and numbers and percentages for categorical variables. The medians were compared using the Mann-Whitney U test. The main outcome variables in the analysis were undernutrition (ie, underweight, stunting, and wasting) versus no undernutrition. The prevalence of undernutrition was calculated. Age was stratified at 2 years because of the known increased risk of undernutrition as children reach their second year of life. ${ }^{30}$ Potential risk factors of undernutrition were tested for significance in a bivariate logistic regression. Variables that achieved a significance level of 0.1 were considered eligible for multivariate logistic regression analysis. For all analyses, $P$-values less than 0.05 were considered statistically significant. Statistical analysis was done using SPSS software (v 16).

\section{Results}

A total of 192 HIV-infected children were seen within the study period but only 182 satisfied the inclusion criteria. Excluded were ten children with incomplete/missing data of interest. Table 1 is the age, sex and undernutrition distribution 
Table 4 Prevalence and risk factors of stunting among subjects aged under 5 years old

\begin{tabular}{|c|c|c|c|c|c|c|c|c|}
\hline \multirow[t]{2}{*}{ Clinical variable } & \multirow{2}{*}{$\begin{array}{l}\text { Stunted } \\
\text { (HAZ-score }<-2 S D) \\
\text { N (\%) }\end{array}$} & \multirow{2}{*}{$\begin{array}{l}\text { Not stunted } \\
\text { (HAZ-score } \geq-2 S D \text { ) } \\
\text { N (\%) }\end{array}$} & \multicolumn{3}{|c|}{ Bivariate analysis } & \multicolumn{3}{|c|}{ Multivariate analysis } \\
\hline & & & $\overline{\text { OR }}$ & $95 \% \mathrm{Cl}$ & $P$-value & AOR & $95 \% \mathrm{Cl}$ & $P$-value \\
\hline \multicolumn{9}{|l|}{ Demography } \\
\hline \multicolumn{9}{|l|}{ Age (months) } \\
\hline Median & 2.00 & 3.00 & - & - & 0.053 & - & - & - \\
\hline $0-23$ & $20(20.2)$ & $14(16.9)$ & 1.248 & $0.586-2.656$ & 0.565 & - & - & - \\
\hline $24-59$ (ref) & $79(79.8)$ & $69(83.1)$ & & & & & & \\
\hline \multicolumn{9}{|l|}{ Sex } \\
\hline Male & $5 \mid(5 \mid .5)$ & $37(44.6)$ & 1.321 & $0.736-2.372$ & $0.35 \mathrm{I}$ & - & - & - \\
\hline Female (ref) & $48(48.5)$ & $46(55.4)$ & & & & & & \\
\hline \multicolumn{9}{|l|}{ Orphanhood } \\
\hline Yes & $27(27.3)$ & $22(26.5)$ & 1.040 & $0.538-2.008$ & 0.908 & - & - & - \\
\hline No (ref) & $72(72.7)$ & $61(73.5)$ & & & & & & \\
\hline \multicolumn{9}{|c|}{ Family/socioeconomic factors } \\
\hline Mode of HIV transmission & & $66(79.5)$ & & & & & & \\
\hline Vertical & $96(97.0)$ & $17(20.5)$ & 8.242 & $2.322-29.255$ & 0.000 & 0.000 & - & 0.999 \\
\hline Blood transfusion (ref) & $3(3.0)$ & $66(79.5)$ & & & & & & \\
\hline \multicolumn{9}{|l|}{ Mode of infant feeding } \\
\hline EBMS & $68(68.7)$ & $13(15.7)$ & - & - & 0.000 & 0.136 & $0.032-0.585$ & 0.007 \\
\hline MF & $15(15.2)$ & $63(75.9)$ & & & & 2.165 & $0.48 I-9.758$ & 0.314 \\
\hline EBF (ref) & $16(16.2)$ & $7(8.4)$ & & & & & & \\
\hline \multicolumn{9}{|l|}{ Caregivers' HIV Status } \\
\hline Infected & $96(97.0)$ & $68(81.9)$ & 7.059 & $1.967-25.335$ & 0.001 & - & - & - \\
\hline Not infected (ref) & $3(3.0)$ & $15(18.1)$ & & & & & & \\
\hline \multicolumn{9}{|l|}{ Caregiver on HAART } \\
\hline No & $21(21.9)$ & $7(10.3)$ & 2.440 & $0.973-6.121$ & 0.052 & 0.604 & $0.166-2.199$ & 0.445 \\
\hline Yes (ref) & $75(78.1)$ & $61(89.7)$ & & & & & & \\
\hline \multicolumn{9}{|l|}{ Marital status of caregiver } \\
\hline Without partner & $15(15.2)$ & $10(12.0)$ & 1.304 & $0.552-3.079$ & 0.545 & - & - & - \\
\hline With a partner (ref) & $84(84.8)$ & $73(88.0)$ & & & & & & \\
\hline \multicolumn{9}{|c|}{ Number of people in the household } \\
\hline$>5$ & $53(53.5)$ & $43(5 \mid .8)$ & 1.072 & $0.598-1.922$ & 0.816 & - & - & - \\
\hline$\leq 5$ (ref) & $46(46.5)$ & $40(48.2)$ & & & & & & \\
\hline \multicolumn{9}{|l|}{ Place of residence } \\
\hline Urban & $14(14.1)$ & $10(12.0)$ & 1.202 & $0.504-2.869$ & 0.678 & - & - & - \\
\hline Rural (ref) & $85(85.9)$ & $73(88.0)$ & & & & & & \\
\hline Clinical/laboratory find & & & & & & & & \\
\hline WHO clinical staging & & & & & & & & \\
\hline 3 and 4 & $29(29.3)$ & $20(24.1)$ & 1.305 & $0.672-2.534$ & 0.431 & - & - & - \\
\hline 1 and 2 (ref) & 70 (70.7) & $63(75.9)$ & & & & & & \\
\hline CD4 count & & & & & & & & \\
\hline Median (IQR) & $55.50(268.0-938.75)$ & - & - & - & 0.066 & - & - & - \\
\hline$\leq 200$ & $20(20.2)$ & $12(14.5)$ & 1.498 & $0.684-3.281$ & 0.311 & - & - & - \\
\hline$>200$ (ref) & $79(79.8)$ & 71 (85.5) & & & & & & \\
\hline Viral load (copies/mL) & & & & & & & & \\
\hline Median log 10 (IQR) & $4.66(3.716-5.315)$ & - & - & - & 0.149 & - & - & - \\
\hline$>10,000$ & $72(72.7)$ & $52(62.7)$ & 1.590 & $0.849-2.977$ & 0.146 & - & - & - \\
\hline$<10,000$ (ref) & $27(27.3)$ & $31(37.3)$ & & & & & & \\
\hline Hepatitis B surface antigen & & & & & & & & \\
\hline Yes & $9(9.1)$ & $9(10.8)$ & 0.822 & $0.311-2.177$ & 0.693 & - & - & - \\
\hline No (ref) & $90(90.9)$ & $74(89.2)$ & & & & & & \\
\hline Hepatitis $C$ antibodies & & & & & & & & \\
\hline Yes & $3(3.0)$ & $2(2.4)$ & 1.250 & $0.204-7.666$ & 1.000 & - & - & - \\
\hline No (ref) & $96(97.00)$ & $80(97.6)$ & & & & & & \\
\hline Diagnosed comorbiditi & s/opportunistic infectio & & & & & & & \\
\hline Tuberculosis & & & 1.127 & $0.545-2.334$ & 0.747 & - & - & - \\
\hline Yes & $2 \mid(2 \mid .2)$ & $16(19.3)$ & & & & & & \\
\hline No (ref) & $78(78.8)$ & $67(80.7)$ & & & & & & \\
\hline
\end{tabular}


Table 4 (Continued)

\begin{tabular}{|c|c|c|c|c|c|c|c|c|}
\hline \multirow[t]{2}{*}{ Clinical variable } & \multirow{2}{*}{$\begin{array}{l}\text { Stunted } \\
\text { (HAZ-score }<-2 S D) \\
\text { N (\%) }\end{array}$} & \multirow{2}{*}{$\begin{array}{l}\text { Not stunted } \\
\text { (HAZ-score } \geq-2 S D) \\
\text { N (\%) }\end{array}$} & \multicolumn{3}{|c|}{ Bivariate analysis } & \multicolumn{3}{|c|}{ Multivariate analysis } \\
\hline & & & OR & $95 \% \mathrm{Cl}$ & $P$-value & AOR & $95 \% \mathrm{Cl}$ & $P$-value \\
\hline \multicolumn{9}{|c|}{ Oropharyngeal candidiasis } \\
\hline Yes & $9(9.1)$ & $9(10.8)$ & 0.822 & $0.311-2.177$ & 0.693 & - & - & - \\
\hline No (ref) & $90(90.9)$ & $74(89.2)$ & & & & & & \\
\hline \multicolumn{9}{|c|}{ Esophageal candidiasis } \\
\hline Yes & $4(4.0)$ & $\mathrm{I}(\mathrm{I} .2)$ & 3.453 & $0.378-31.509$ & 0.244 & - & - & - \\
\hline No (ref) & $95(96.0)$ & $82(98.8)$ & & & & & & \\
\hline \multicolumn{9}{|l|}{ Diarrheal disease } \\
\hline Yes & $5(5.1)$ & $2(2.4)$ & 2.154 & $0.407-11.404$ & 0.356 & - & - & - \\
\hline No (ref) & $94(94.9)$ & $81(97.6)$ & & & & & & \\
\hline \multicolumn{9}{|l|}{ Sepsis } \\
\hline Yes & $3(3.0)$ & $6(7.2)$ & 0.401 & $0.097-1.656$ & 0.193 & - & - & - \\
\hline No (ref) & $96(97.0)$ & $77(92.8)$ & & & & & & \\
\hline \multicolumn{9}{|l|}{ Sickle-cell disease } \\
\hline Yes & $4(4.0)$ & $5(6.0)$ & 0.657 & $0.17 I-2.530$ & 0.539 & - & - & - \\
\hline No (ref) & $95(96.0)$ & $78(94.0)$ & & & & & & \\
\hline \multicolumn{9}{|l|}{ Pneumonia } \\
\hline Yes & $4(4.0)$ & $4(4.8)$ & 0.832 & $0.20 \mathrm{I}-3.432$ & 0.798 & - & - & - \\
\hline No (ref) & $95(96.0)$ & $79(95.2)$ & & & & & & \\
\hline \multicolumn{9}{|l|}{ Anemia $(<8 \mathrm{~g} / \mathrm{dL})$} \\
\hline Yes & $62(62.6)$ & $47(56.6)$ & 0.779 & $0.430-1.413$ & $0.4 \mathrm{II}$ & - & - & - \\
\hline No & $37(37.4)$ & $36(43.4)$ & & & & & & \\
\hline
\end{tabular}

Abbreviations: AOR, adjusted odds ratio; $\mathrm{Cl}$, confidence interval; EBF, exclusive breast feeding; EBMS, exclusive breast-milk-substitute feeding; HAART, highly active antiretroviral therapy; IQR, interquartile range; MF, mixed feeding; N, number; OR, odds ratio; Ref, reference group; SD, standard deviation; HAZ-score, height-for-age Z-score; WHO, World Health Organization.

among the 182 subjects. The 182 subjects were between 6 weeks and 59 months of age, with a median age of 36 months and interquartile range of 24-48 months.

There were 88 males (M) and 94 females (F) with an $\mathrm{M}: \mathrm{F}$ ratio of 1.0:1.1. The prevalence of undernutrition was $12.1 \%$ (ie, 22/182), 33.5\% (61/182), and 54.4\% (99/182) for underweight, wasting, and stunting, respectively. Sixtynine subjects $(37.9 \%)$ did not have any form of undernutrition. Table 2 shows the prevalence and risk factors of underweight among the subjects.

In multivariate analyses, only the subject's sex, whether the caregivers of the child were on ART, and the presence of coinfection with tuberculosis were independently associated with underweight. The trend was such that female subjects were 0.292 less likely to be underweight (AOR $0.292,95 \%$ CI $0.104-0.820, P=0.019$ ) and when the child came from a household in which their caregiver was on ART, the child was also significantly protected against underweight (AOR 0.190, 95\% CI 0.039-0.925, $P=0.04$ ). Also, the absence of tuberculosis in the child protected against underweight (AOR 0.034, 95\% CI 0.003-0.357, $P=0.005$ ).

Table 3 reveals the prevalence and risk factors of wasting among the subjects. No variable was found to be significantly associated with wasting in multivariate analyses.
Table 4 is the prevalence and risk factors of stunting among the subjects. Only the mode of early infant feeding was significantly associated with stunting in the multivariate analyses. EBF was found to be significantly protective against stunting compared with EBMS (AOR 0.136, 95\% CI $0.032-0.585, P=0.007)$, although fewer subjects practiced EBF than EBMS (12.6\% [13/182] vs 44.6\% [81/182]).

\section{Discussion}

In the present study, among the HIV-infected ART-naïve children, the prevalence of undernutrition was $12.1 \%$, $33.5 \%$, and $54.4 \%$ for underweight, wasting, and stunting, respectively. The prevalence of undernutrition in this study was lower than the prevalence of $58.6 \%$ and $48.6 \%$ for underweight and stunting, respectively, but was higher than the $31.4 \%$ for wasting, among similarly HIV-infected ART-naïve children in Ile-Ife, Osun State, in the South Western region of Nigeria. ${ }^{17}$ Furthermore, the prevalence of undernutrition in this study was lower than the $63 \%$ and $58 \%$ reported for underweight and stunting, respectively, but higher than the $16 \%$ reported for wasting among HIV-infected ART-naïve Indian children. ${ }^{31}$

Differences in prenatal growth patterns, varying food supplementation/socioeconomic conditions, and differences in disease manifestation due to differing HIV subtypes among 
the infected children in the different study settings could explain the dissimilarities observed between our study and the studies of others. ${ }^{17,31}$

"Underweight" (weight for age) is a composite index of weight-for-height and height-for-age. It does not distinguish between acute malnutrition (wasting) and chronic malnutrition (stunting), but it is a good overall indicator of a population's nutritional health. ${ }^{32}$ In the present study, female HIV-infected subjects were 0.292 less likely to be underweight. In a Tanzanian study, ${ }^{33}$ male children born to HIV-infected women were $28 \%$ more likely to become underweight in comparison to females. Similarly, in a study from Andhra Pradesh, India, male children in the general population were twice as likely and $50 \%$ more likely than females to be wasted and underweight, respectively, after controlling for multiple other risk factors in the respective analyses. ${ }^{34}$ McDonald et a ${ }^{33}$ had earlier suggested a preferential treatment toward girls in terms of health care-seeking behavior or dietary consumption. Contrarywise, in our own cultural setting, preferential treatment by the parents argues more in favor of a male child, since there is a strong societal preference for a male child and this makes the association found in our study more difficult to explain.

Also, the present study reveals that when the HIV-infected caregivers of our subjects were on HAART, a significant protection against underweight in the child was seen. A combination of reduced earnings due to frailty, reduced savings and assets, and high adult mortality causes food insecurity in HIV households, and food insecurity increases the risk of malnutrition among children in the household. ${ }^{35,36}$ However, following the commencement of ART in caregivers, employment and income have been found to increase, thereby improving food security and the nutritional status of all the household members. ${ }^{35-37}$ This observation may also explain our finding of the protective effect against underweight seen among children of caregivers who had been initiated on ART in this study.

Not surprisingly, absence of tuberculosis in the child was found to protect against underweight in the present study. Coinfection with HIV and tuberculosis is an extremely common problem, and, in areas of high prevalence, it is the most common coinfection in HIV-positive children. ${ }^{35}$ HIV and tuberculosis pathogens interact, resulting in weight loss, which is the presenting feature in almost $50 \%$ of cases of tuberculosis, and persistent anorexia, a feature in approximately one-quarter of cases, also contributes to undernutrition. ${ }^{38}$ Furthermore, malnutrition is a risk factor for the acquisition of primary tuberculosis infection, as well as progression to active disease. Unfortunately, most health facilities in Nigeria, including our own, have not adopted the use of isoniazid preventive therapy for tuberculosis, while intensified case-finding of tuberculosis and infection control for tuberculosis, the other two components of the "three Is" for HIV/tuberculosis coinfection control, are still suboptimal in Nigeria.

Stunting is an indicator of a cumulative effect of chronic malnutrition $^{32}$ and EBF was found to be significantly protective against stunting compared with formula feeding in the present study. Similarly, an Eastern Uganda study reported that children on EBF were less likely to be stunted. ${ }^{39}$ However, fewer than $12.6 \%$ of our subjects practiced EBF compared with $44.6 \%$ who practiced EBMS. In our ART program and during the period of study, infant formula was provided free of cost to the mothers, and may thus explain the higher preference for this mode of infant feeding, even after the provision of the necessary information to guide mothers' decisions. Breastfeeding has been shown to help prevent growth faltering and stunting, particularly as it reduces the risk of infectious diseases, ${ }^{40-44}$ which are the important determinants of stunting. ${ }^{40,45}$ Although breastfed children may still become stunted if they do not receive an adequate quantity and quality of complementary foods from the age of 6 months onward, ${ }^{46}$ the risk may be less among early exclusively breastfed infants in the first 6 months of life, as seen in the present study.

\section{Limitations of the study}

Although we considered a comprehensive set of variables in our analyses, residual confounding covariates that were unmeasured and/or missing in the records were not tested. Being a retrospective study, these covariates were not given attention at the point of recruitment into our ART program and we were therefore constrained in this regard. We would have loved to test these other a priori covariates of child undernutrition, including child-level factors such as gestational age and birth weight; maternal-level factors such as maternal size, age, and education; household-level factors such as food insecurity, pattern of complementary feeding, and socioeconomic status; and community-level factors such as sanitation and environmental factors. ${ }^{47-49}$ Also, parasitic infestations that impact negatively on nutritional status and growth could not be determined, because stool microscopy is not a routine investigation for our subjects on enrollment into our ART program.

Moreover, being a cross-sectional study, establishing a cause and effect relationship between the tested variables and undernutrition was limited. 
In addition, the lack of significant association in our multivariate analyses between wasting and the tested variables may be due to the small sample size of the study, and it is also possible that these variables may have been present but had resolved in many more subjects before enrollment into our ART program, and, therefore, the absence of statistical significance has to be considered cautiously.

Finally, the lack of data on undernutrition in a comparable group of HIV-negative children in the same setting during the same period of study limits interpretation of the results of this study.

\section{Conclusion}

Despite the outlined limitations, the present study shows that the prevalence of undernutrition among HIV-infected ARTnaïve under-5-year-olds is high, at 12.1\%, 33.5\%, and 54.4\% for underweight, wasting, and stunting, respectively. Multivariate analyses showed that being female, having a caregiver on ART, and the absence of tuberculosis in the child are protective against underweight, and that when subjects had EBF in the first 6 months of life, protection against stunting was seen. Therefore, programmatic intervention should be well guided toward the early initiation of HAART among eligible HIV-infected caregivers and the promotion of the "three Is" of HIV/tuberculosis coinfection control: isoniazid preventive therapy, intensified case-finding of tuberculosis, and the infection control for tuberculosis. Also, the importance of EBF in reducing the prevalence of undernutrition and its consequent morbidity and mortality among our HIV-infected ART-naïve children cannot be overemphasized.

\section{Acknowledgments}

This work was funded in part by the US Department of Health and Human Services, Health Resources and Services Administration (U51HA02522) and the Centers for Disease Control and Prevention through APIN (PS001058).

\section{Author contributions}

This work was carried out by both authors in collaboration. Emmanuel Ademola Anigilaje designed the study, wrote the protocol and collated the data, performed and reviewed the statistical analysis, did literature searches, wrote the first draft of the manuscript and also critically reviewed it for intellectual content. Ayodotun Olutola was involved in the design of the study, reviewed the statistical analyses, provided literature reviews, and critically reviewed the article for intellectual content. Both authors agreed to the final version of the manuscript submitted for publication.

\section{Disclosure}

The authors report no conflicts of interest in this work.

\section{References}

1. Federal Ministry of Health Nigeria Department of Health National AIDS/STI Control Programme. 2010 National HIV Sero-Prevalence Sentinel Survey. Abuja: Federal Ministry of Health Nigeria; 2010. Available from: http://www.nigeria-aids.org/documents/2010_National\%20 HIV\%20Sero\%20Prevalence\%20Sentinel\%20Survey.pdf. Accessed February 11, 2015.

2. Ojoawo A, Dairo O, Ojetunde A. City Profile of HIV/AIDS in the City of Makurdi, Nigeria. Ibadan: Development Policy Centre; 2005. Available from: http://mirror.unhabitat.org/downloads/docs/4058_99135_ Makurdi.pdf. Accessed February 11, 2015.

3. Joint United Nations Programme on HIV/AIDS (UNAIDS). 2013 Progress Report on the Global Plan: Towards the Elimination of New HIV Infections among Children by 2015 and Keeping Their Mothers Alive. Geneva: UNAIDS; 2013. Available from: http://www.unaids.org/ sites/default/files/media_asset/20130625_progress_global_plan_en_0. pdf. Accessed February 11, 2015.

4. Anígilájé EA, Dabit OJ, Ageda B, Hwande S, Bitto TT. The prevalence and predictors of HIV infection among children of mothers who missed prevention of mother to child transmission of HIV interventions in Makurdi, Nigeria. J AIDS Clin Res. 2013;4:249.

5. National Population Commission (NPC; Nigeria), ICF Macro. Nigeria: Demographic and Health Survey Abuja: NPC, ICF Macro; 2008. Available from: http://dhsprogram.com/pubs/pdf/fr222/fr222.pdf. Accessed February 11, 2015.

6. United States Embassy in Nigeria Economic Section. Nigeria HIV Fact Sheet. United States Embassy in Nigeria; 2011. Available from: http://photos.state.gov/libraries/nigeria/487468/pdfs/DecemberHIVFactSheet.pdf. Accessed February 11, 2015.

7. Berhane R, Bagenda D, Marum L, et al. Growth failure as a prognostic indicator of mortality in pediatric HIV infection. Pediatrics. 1997; 100(1):E7.

8. Chantry CJ, Byrd RS, Englund JA, Baker CJ, McKinney RE Jr; Pediatric AIDS Clinical Trials Group Protocol 152 Study Team. Growth, survival and viral load in symptomatic childhood human immunodeficiency virus infection. Pediatr Infect Dis J. 2003;22(12):1033-1039.

9. Fergusson P, Tomkins A. HIV prevalence and mortality among children undergoing treatment for severe acute malnutrition in sub-Saharan Africa: a systematic review and meta-analysis. Trans $R$ Soc Trop Med Hyg. 2009;103(6):541-548.

10. Musoke PM, Mudiope P, Barlow-Mosha LN, et al. Growth, immune and viral responses in HIV infected African children receiving highly active antiretroviral therapy: a prospective cohort study. BMC Pediatr. 2010;10:56.

11. World Health Organization (WHO). World Health Statistics 2009. Geneva: WHO; 2009. Available from: http://www.who.int/gho/ publications/world_health_statistics/EN_WHS09_Full.pdf. Accessed February 11, 2015.

12. Rajasekaran S, Jeyaseelan L, Ravichandran N, Gomathi C, Thara F, Chandrasekar C. Efficacy of antiretroviral therapy program in children in India: prognostic factors and survival analysis. $J$ Trop Pediatr. 2009;55(4):225-232.

13. Arpadi SM. Growth failure in children with HIV infection. J Acquir Immune Defic Syndr. 2000;25 Suppl 1:S37-S42.

14. Miller TL. Nutritional aspects of HIV-infected children receiving highly active antiretroviral therapy. AIDS. 2003;17 Suppl 1:S130-S140.

15. Arpadi SM, Cuff PA, Kotler DP, et al. Growth velocity, fat-free mass and energy intake are inversely related to viral load in HIV-infected children. J Nutr. 2000;130(10):2498-2502.

16. Ezeonwu BU, Ikefuna AN, Oguonu T, Okafor HU. Prevalence of hematological abnormalities and malnutrition in HIV-infected under five children in Enugu. Niger J Clin Pract. 2014;17(3):303-308.

17. Anyabolu HC, Adejuyigbe EA, Adeodu OO. Undernutrition and anaemia among HAART-naïve HIV infected children in Ile-Ife, Nigeria: a case-controlled, hospital based study. Pan Afr Med J. 2014;18:77. 
18. Obiagwu PN, Hassan-Hanga F, Ibrahim M. Pediatric HIV in Kano, Nigeria. Niger J Clin Pract. 2013;16(4):521-525.

19. Chiabi A, Lebela J, Kobela M, Mbuagbaw L, Obama MT, Ekoe T. The frequency and magnitude of growth failure in a group of HIV-infected children in Cameroon. Pan Afr Med J. 2012;11:15.

20. Shet A, Mehta S, Rajagopalan N, et al. Anemia and growth failure among HIV-infected children in India: a retrospective analysis. $B M C$ Pediatr. 2009;9:37.

21. Orphan Nutrition. Impact of malnutrition on health and development [web page on the Internet]. Alexandria, VA: Joint Council. Available from: http://www.orphannutrition.org/understanding-malnutrition/impact-ofmalnutrition-on-health-and-development/. Accessed February 2, 2014.

22. Banerjee T, Pensi T, Banerjee D, Grover G. Impact of HAART on survival, weight gain and resting energy expenditure in HIV-1-infected children in India. Ann Trop Paediatr. 2010;30(1):27-37.

23. Sutcliffe CG, van Dijk JH, Munsanje B, et al. Weight and height Z-scores improve after initiating ART among HIV-infected children in rural Zambia: a cohort study. BMC Infect Dis. 2011;11:54.

24. Sunguya BF, Poudel KC, Otsuka K, et al. Undernutrition among HIVpositive children in Dar es Salaam, Tanzania: antiretroviral therapy alone is not enough. BMC Public Health. 2011;11:869.

25. World Health Organization. Global Health Observatory Data Repository. Pediatric antiretroviral therapy coverage data by country: 2012. Available from: http://apps.who.int/gho/data/node.main.PEDART?lang=en. Accessed March 03, 2015.

26. Federal Ministry of Health Nigeria. National Guidelines for Paediatric HIV and AIDS Treatment and Care. Abuja: Federal Ministry of Health Nigeria; 2007. Available from: http://www.who.int/hiv/amds/Nigeria paediatric_2007.pdf. Accessed February 11, 2015.

27. Gaillard P, Piwoz EG, Farley TM. Collection of standardized information on infant feeding in the context of mother-to-child transmission of HIV. Stat Med. 2001;20(23):3525-3537.

28. WHO Anthro [computer software]. V 3.2.2. Geneva: WHO; 2011. Available from: http://www.who.int/childgrowth/software/en/. Accessed February 11, 2015

29. WHO. Pocket Book of Hospital Care for Children: Guidelines for the Management of Common Childhood Illnesses. 2nd ed. WHO: Geneva; 2013. Available from: http://apps.who.int/iris/bitstream/10665/81170/ 1/9789241548373_eng.pdf. Accessed February 11, 2015.

30. Chavez A, Martinez C, Soberanes B. The effect of malnutrition on human development: a 24-year study of well-nourished and malnourished children living in a poor Mexican village. In: Goodman AH, Dufour DK, Pelto GH, editors. Nutritional Anthropology: Biocultural Perspectives on Food and Nutrition. San Francisco, CA: Mayfield; 2000:234-268.

31. Padmapriyadarsini C, Pooranagangadevi N, Chandrasekaran K, et al. Prevalence of Underweight, Stunting, and Wasting among Children Infected with Human Immunodeficiency Virus in South India. Int $J$ Pediatr. 2009;2009:837627.

32. NPC, ICF International. Nigeria Demographic and Health Survey 2013. Abuja and Rockville, MD: NPC and ICF International. Available from: http://dhsprogram.com/pubs/pdf/FR293/FR293.pdf. Accessed February 11, 2015.

33. McDonald CM, Kupka R, Manji KP, et al. Predictors of stunting, wasting and underweight among Tanzanian children born to HIV-infected women. Eur J Clin Nutr. 2012;66(11):1265-1276.
34. Meshram II, Laxmaiah A, Gal Reddy Ch, Ravindranath M, Venkaiah K, Brahmam GN. Prevalence of under-nutrition and its correlates among under 3 year-old children in rural areas of Andhra Pradesh, India. Ann Hum Biol. 2011;38(1):93-101.

35. Rose AM, Hall CS, Martinez-Alier N. Aetiology and management of malnutrition in HIV-positive children. Arch Dis Child. 2014;99(6): $546-551$.

36. Bor J, Tanser F, Newell ML, Bärnighausen T. In a study of a population cohort in South Africa, HIV patients on antiretrovirals had nearly full recovery of employment. Health Aff (Millwood). 2012;31(7): $1459-1469$.

37. Thirumurthy H, Jafri A, Srinivas G, et al. Two-year impacts on employment and income among adults receiving antiretroviral therapy in Tamil Nadu, India: a cohort study. AIDS. 2011;25(2):239-246.

38. Miller LG, Asch SM, Yu EI, Knowles L, Gelberg L, Davidson P. A population-based survey of tuberculosis symptoms: how atypical are atypical presentations? Clin Infect Dis. 2000;30(2):293-299.

39. Engebretsen IM, Tylleskär T, Wamani H, Karamagi C, Tumwine JK. Determinants of infant growth in Eastern Uganda: a community-based cross-sectional study. BMC Public Health. 2008;8:418.

40. United Nations Children's Fund (UNICEF). Scientific Rationale: Benefits of Breastfeeding; 2012. New York, NY: UNICEF; 2012. Available from: http://www.unicef.org/nutrition/files/Scientific_rationale_for_ benefits_of_breasfteeding.pdf. Accessed February 2, 2014.

41. López-Alarcón M, Villalpando S, Fajardo A. Breast-feeding lowers the frequency and duration of acute respiratory infection and diarrhea in infants under six months of age. J Nutr. 1997;127(3):436-443.

42. Froozani MD, Permehzadeh K, Motlagh AR, Golestan B. Effect of breastfeeding education on the feeding pattern and health of infants in their first 4 months in the Islamic Republic of Iran. Bull World Health Organ. 1999;77(5):381-385.

43. Arifeen SE, Black RE, Caulfield LE, Antelman G, Baqui AH. Determinants of infant growth in the slums of Dhaka: size and maturity at birth, breastfeeding and morbidity. Eur J Clin Nutr. 2001;55(3): $167-178$.

44. Kramer MS, Guo T, Platt RW, et al; PROBIT Study Group. Breastfeeding and infant growth: biology or bias? Pediatrics. 2002;110(2 Pt 1): 343-347.

45. Scrimshaw NS, Taylor CE, Gordon JE. Interactions of nutrition and infection. Monogr Ser World Health Organ. 1968;57:3-329.

46. Black RE, Allen LH, Bhutta ZA, et al; Maternal and Child Undernutrition Study Group. Maternal and child undernutrition: global and regional exposures and health consequences. Lancet. 2008;371(9608): $243-260$.

47. Hien NN, Kam S. Nutritional status and the characteristics related to malnutrition in children under five years of age in Nghean, Vietnam. J Prev Med Public Health. 2008;41(4):232-240.

48. Linnemayr S, Alderman H, Ka A. Determinants of malnutrition in Senegal: individual, household, community variables, and their interaction. Econ Hum Biol. 2008;6(2):252-263.

49. Pongou R, Ezzati M, Salomon JA. Household and community socioeconomic and environmental determinants of child nutritional status in Cameroon. BMC Public Health. 2006;6:98.
International Journal of General Medicine

\section{Publish your work in this journal}

The International Journal of General Medicine is an international, peer-reviewed open-access journal that focuses on general and internal medicine, pathogenesis, epidemiology, diagnosis, monitoring and treatment protocols. The journal is characterized by the rapid reporting of reviews, original research and clinical studies across all disease areas.

\section{Dovepress}

A key focus is the elucidation of disease processes and management protocols resulting in improved outcomes for the patient.The manuscript management system is completely online and includes a very quick and fair peer-review system. Visit http://www.dovepress.com/ testimonials.php to read real quotes from published authors. 